\title{
Realtime 3D stress measurement in curing epoxy packaging
}

Richter, Jacob; Hyldgård, A.; Birkelund, Karen; Hansen, Ole; Thomsen, Erik Vilain

Published in:

International Solid-State Sensors, Actuators and Microsystems Conference, 2007. TRANSDUCERS 2007.

Link to article, DOI:

10.1109/SENSOR.2007.4300575

Publication date:

2007

Document Version

Publisher's PDF, also known as Version of record

Link back to DTU Orbit

Citation (APA):

Richter, J., Hyldgård, A., Birkelund, K., Hansen, O., \& Thomsen, E. V. (2007). Realtime 3D stress measurement in curing epoxy packaging. In International Solid-State Sensors, Actuators and Microsystems Conference, 2007. TRANSDUCERS 2007. (pp. 2083-2084). IEEE. https://doi.org/10.1109/SENSOR.2007.4300575

\section{General rights}

Copyright and moral rights for the publications made accessible in the public portal are retained by the authors and/or other copyright owners and it is a condition of accessing publications that users recognise and abide by the legal requirements associated with these rights.

- Users may download and print one copy of any publication from the public portal for the purpose of private study or research.

- You may not further distribute the material or use it for any profit-making activity or commercial gain

- You may freely distribute the URL identifying the publication in the public portal 


\title{
REALTIME 3D STRESS MEASUREMENT IN CURING EPOXY PACKAGING
}

\author{
J. Richter ${ }^{1}$, A. Hyldgård ${ }^{1}$, K. Birkelund ${ }^{1}$, O. Hansen ${ }^{1}$ and E.V. Thomsen ${ }^{1}$ \\ ${ }^{1}$ Department of Micro and Nanotechnology, Technical University of Denmark, \\ Kgs Lyngby DK-2800, DENMARK \\ (Tel : +45-4525-5700; E-mail: jar@mic.dtu.dk)
}

\begin{abstract}
This paper presents a novel method to characterize stress in microsystem packaging. A circular $p$-type piezoresistor is implemented on a (001) silicon chip. We use the circular stress sensor to determine the packaging induced stress in a polystyrene tube filled with epoxy. The epoxy curing process is monitored by stress measurements. From the stress measurements we conclude that the epoxy cures in 8 hours at room temperature. We find the difference in in-plane normal stresses to be $\sigma_{x x}-\sigma_{y y}=6.7 \mathrm{MPa}$ and $\left(\sigma_{x x}+\sigma_{y y}-0.4 \sigma_{z z}\right)=232 \mathrm{MPa}$.
\end{abstract}

Keywords: stress sensor, epoxy, packaging.

\section{INTRODUCTION}

Packaging and protection of a MEMS device is the last step before commercialization. It is a difficult task to protect the device and not affect the performance of the device at the same time since the electrical performance can be highly influenced by packaging induced stress. This induced stress can be measured by a dedicated stress sensor. In ref. [1] a CMOS integrated stress sensor for packaged integrated circuit dies is presented, and ref. [2] shows a van der Pauw structure used for stress sensing.

In this paper we present a method to determine stress in microsystem packaging. We measure the stress distribution in an epoxy package while the epoxy is curing. The packaging concept is otherwise used to protect chip and electronics in a multisensor system developed for fisheries research [3]. This method makes complex stress analysis possible in microscale packaging.

We have designed a (001) p-type circular piezoresistor to determine the stress distribution in the package. The design is inspired by the work of Bartholomeyczik [4] and Steiner [5]. The device is shown in Figure 1.

\section{THEORY}

The chip consists of one circular piezoresistor. A conceptual drawing of the device is shown in Figure 2. We direct the current, $I(\phi)$, at an angle $\phi$

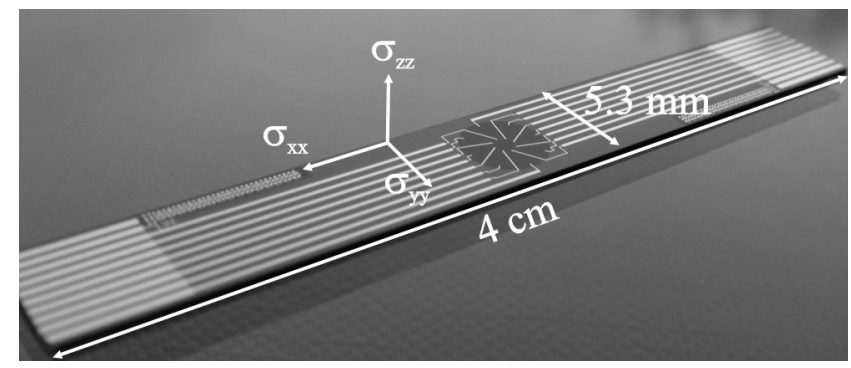

Figure 1. Presentation of stress sensor. The chip is cut along the [110] direction. In the centre region of the chip a single circular p-type resistor is implemented. A current is forced through the resistor by contacts near the perimeter and 8 contacts placed in a radius of $100 \mu \mathrm{m}$ with respect to the resistor centre measure 4 potential drops.

with respect to the [110] crystal direction by using four outer contacts placed near the resistor perimeter. Thus, in the centre of the circular piezoresistor we spin the current $360^{\circ}$.

The inner contacts are placed in a radius of 100 $\mu \mathrm{m}$ with respect to the centre of the circular piezoresistor. The radius of the resistor is 1700 $\mu \mathrm{m}$, thus we assume a uniform current distribution in the region of the inner contacts.

The potential drop, $V_{\mathrm{i}}=V_{\mathrm{ia}}-V_{\mathrm{ib}}$, is measured across the inner contacts where $\mathrm{i}=1,2,3,4$. Thus, for each current direction four potential drops are measured. To a first order approximation the relative change, $\Delta V_{\mathrm{i}} / V_{\mathrm{i}, 0}$, is equal to a linear combination of the directional stress components. Defining two fictive voltage drops, $V_{p}$ and $V_{t}$ in Figure 2, as linear combinations of the four 


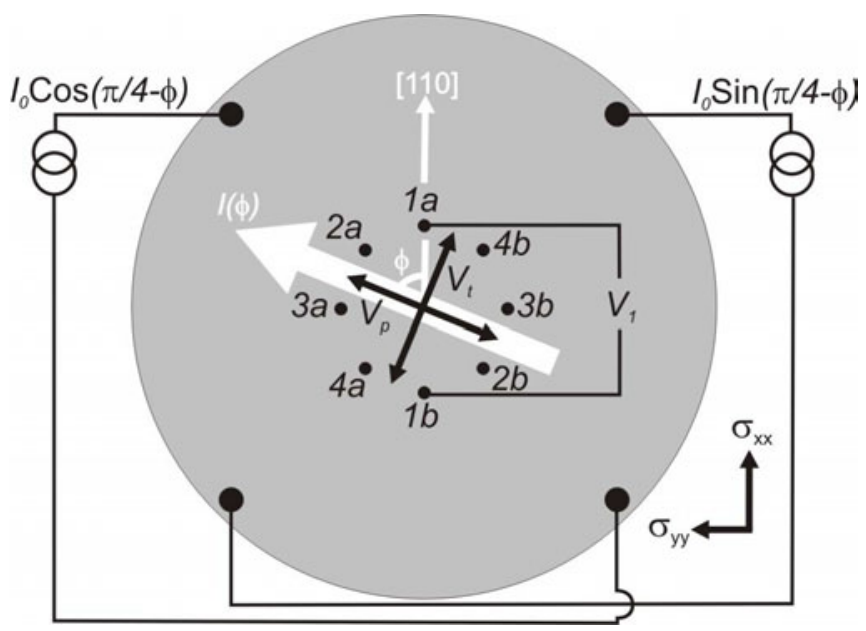

Figure 2. Schematic of the circular piezoresistor. The illustration is not to scale. The resistor radius is $1700 \mu \mathrm{m}$ and the inner contacts are placed in a radius of $100 \mu \mathrm{m}$. The four outer contacts spin the current $360^{\circ}$ in the centre of the resistor. The fictive voltage drops, $V_{p}$ and $V_{t}$, are linear combinations of all measured potential drops, $V_{i}=V_{i a}-V_{i b}$ where $i=1,2,3,4$ as defined in Eq. (1). The potential drop $V_{1}$ is showed as an example.

potential drops we obtain a condensed linear system where the number of current directions determines the number of equations. The fictive voltage drops are defined as

$$
\begin{aligned}
V_{p}= & \frac{1}{2}\left(\cos (\phi) \cdot V_{1}+\cos \left(\phi-\frac{\pi}{4}\right) \cdot V_{2}\right. \\
& \left.+\sin (\phi) \cdot V_{3}+\sin \left(\phi-\frac{\pi}{4}\right) \cdot V_{4}\right) \\
V_{t}= & \frac{1}{2}\left(\sin (\phi) \cdot V_{1}+\sin \left(\phi-\frac{\pi}{4}\right) \cdot V_{2}\right. \\
& \left.-\cos (\phi) \cdot V_{3}-\cos \left(\phi-\frac{\pi}{4}\right) \cdot V_{4}\right) .
\end{aligned}
$$

In terms of the piezocoefficients and stress components we find

$$
\begin{aligned}
\frac{\Delta V_{p}}{V_{p, 0}=} & \frac{\sigma_{x x}}{2}\left(\pi_{11}+\pi_{12}+\pi_{44}\right) \cos (2 \phi) \\
& \frac{\sigma_{y y}}{2}\left(\pi_{11}+\pi_{12}-\pi_{44}\right) \cos (2 \phi) \\
& +\sigma_{z z} \pi_{12}+\sigma_{x y}\left(\pi_{11}-\pi_{12}\right) \sin (2 \phi),
\end{aligned}
$$

$\begin{aligned} \frac{V_{t}}{V_{p, 0}}= & -\frac{\sigma_{x x}}{2} \pi_{44} \sin (2 \phi)+\frac{\sigma_{y y}}{2} \pi_{44} \sin (2 \phi) \\ & +\sigma_{x y}\left(\pi_{11}-\pi_{12}\right) \cos (2 \phi)\end{aligned}$

where $\Delta V_{p}=V_{p}-V_{p, 0}$ is the change in the fictive voltage drop $V_{p}$ and $V_{p, 0}$ is the potential drop at zero stress. The piezocoefficients are represented by $\pi_{11}, \pi_{12}$, and $\pi_{44}$ and $\sigma_{x x}, \sigma_{x y}$, and $\sigma_{y y}$ are the inplane stresses and $\sigma_{z z}$ is the out-of-plane normal stress as sketched in Figure 1.

Using different values of $\phi$ in Eqs. (2) (3) we obtain an equation system with three independent equations and we can extract $\sigma_{x y},\left(\sigma_{x x}-\sigma_{y y}\right)$, and $\left(\sigma_{x x}+\sigma_{y y}+a^{\bullet} \sigma_{z z}\right)$, where $\mathrm{a}=2 \pi_{12} /\left(\pi_{11}+\pi_{12}\right)$. In $p$-type silicon this ratio is approximately $\mathrm{a}=2 \pi_{12} /\left(\pi_{11}+\pi_{12}\right) \approx-0.4[6]$.

\section{EXPERIMENTAL}

The fabrication of the stress sensor chip is performed by conventional silicon microtechnology fabrication. The doping concentration of the piezoresistor layer is approximately $N_{\mathrm{A}}=10^{18} \mathrm{~cm}^{-3}$.

The electrical measurements are performed with four point measurements. This is illustrated in Figure 3. The current is spun in angular steps of $5^{\circ}$. Thus we obtain 72 measurements of each of the four voltage drops, $V_{\mathrm{i}}$.

The sensors are calibrated in a state-of-the-art four point bending fixture prior to packaging. This calibration step is included in order to determine the piezocoefficients of the sensor. The four point bending bridge applies a uniaxial and uniform stress to the centre region of the chip where the circular piezoresistor is located. With this prior calibration step we are able to extract the piezocoefficients $\left(\pi_{11}+\pi_{12}\right)$ and $\pi_{44}$. The four point bending fixture is illustrated in Figure 4.

Next, the chip is placed in a polystyrene tube which is subsequently filled with EpoTek 302-3M two-component epoxy. Figure 5 is a photograph of the packaged chip after curing.

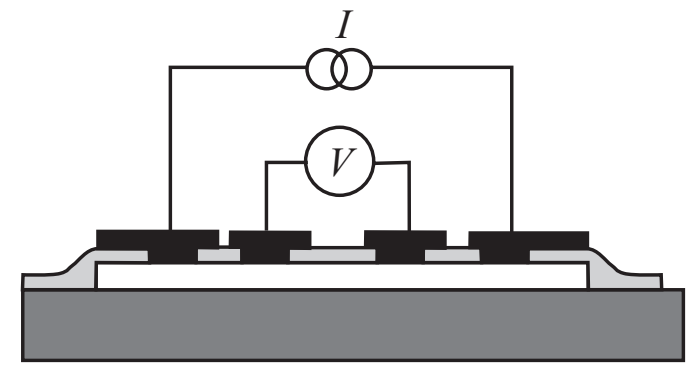

Figure 3. The p-type piezoresistors (white) are implanted on an n-type (001) Si substrate (dark grey). A thermal oxide (light grey) is used for isolation between substrate and metal (black). The resistance of the piezoresistor is measured by a four point measurement. A constant current is forced through the resistor from the outer contacts while a high impedance voltage measurement is performed on the inner contacts. 


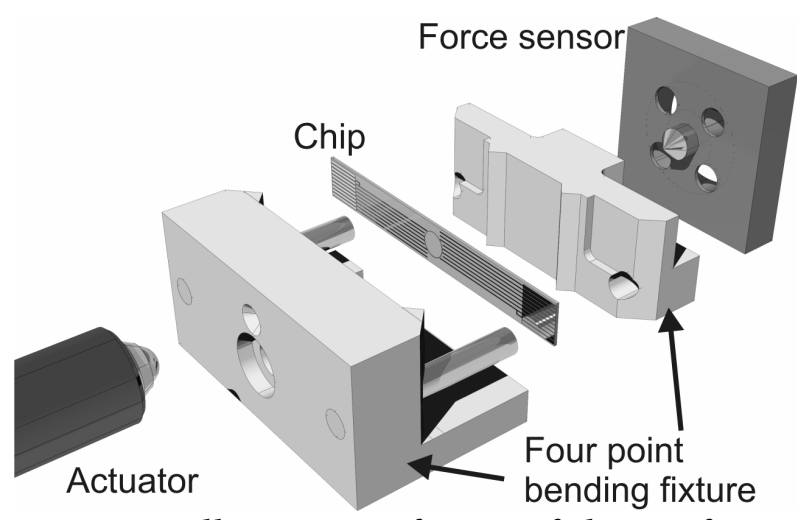

Figure 4. Illustration of state-of-the-art four point bending fixture used for calibration of the stress sensor. The chip is placed in between the two sets of blades and an actuator pushes the two parts together. The force on the chip is measured by a highly sensitive force sensor. The fixture applies a uniaxial and uniform stress to the centre region of the chip. With this calibration step we determine the piezocoefficients.

We perform the same electrical measurements on the chip while the epoxy is curing as performed in the four point bending fixture. The epoxy is cured at room temperature.

\section{RESULTS}

Figure 6 shows the voltage change of the four measured potential drops $\Delta V_{\mathrm{i}}=V_{\mathrm{i}}-V_{\mathrm{i}, 0}$ as a function of current direction, $\phi$ after the epoxy has cured. This figure also includes measurements during calibration where a uniform and uniaxial stress is applied. A linear combination of these four potential drops give us $\Delta V_{p} / V_{p, 0}$ and $\Delta V_{t} / V_{p, 0}$ as defined in Eqs. (2) (3).

During the curing of the epoxy we perform measurements on the stress sensor. Figure 7 shows the change in potential drop $\Delta V_{3} / V_{3,0}$ when the current density vector is directed an angle of $90^{\circ}$ with respect to the [110] direction. The equation for $\Delta V_{3} / V_{3,0}$ for this particular angle is

$$
\begin{aligned}
\left.\frac{\Delta V_{3}}{V_{3,0}}\right|_{\phi=90^{\circ}=} & -\frac{\sigma_{x x}}{2}\left(\pi_{11}+\pi_{12}+\pi_{44}\right) \\
& -\frac{\sigma_{y y}}{2}\left(\pi_{11}+\pi_{12}-\pi_{44}\right) \\
& +\sigma_{z z} \pi_{12}
\end{aligned}
$$

The change in voltage is caused by a change in stress in the chip. This stress is a result of the contraction of epoxy during the curing process.

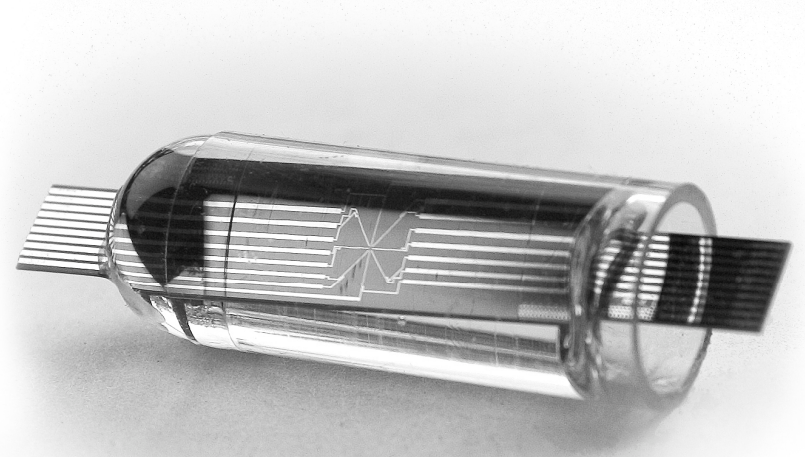

Figure 5. Photograph of the packaged device after curing of the epoxy. The circular piezoresistor is placed in the centre region of the chip. A twocomponent epoxy is filled in a polystyrene tube. The packaging concept allows for direct exposure of one end of a chip while protecting the other end (along with electronics, battery, etc.) from the surroundings.

Figure 7 shows that the epoxy has cured after approximately 8 hours.

From the calculated fictive voltage drops we are able to find the stress distribution in the package. Figure 8 shows a measurement of the fictive voltage drop $\Delta V_{p} / V_{p, 0}$ both when a unaxial stress is applied to the device and when the device is in the package. Previous stress measurements in epoxy packaging [7] have shown a very low $\sigma_{x y}$ value. Thus we assume this value to be equal to zero. From the slope and offset of the linear fit in Figure 8 we find the stress distribution in the package to be $\left(\sigma_{x x}-\sigma_{y y}\right)=6.7 \mathrm{MPa}$, and $\left(\sigma_{x x}+\sigma_{y y^{-}}\right.$ $\left.0.4 \sigma_{z z}\right)=232 \mathrm{MPa}$. The value for $\left(\sigma_{x x}-\sigma_{y y}\right)$ can also be found by using the extracted values of $\Delta V_{t} / V_{p, 0}$.

For all equations $\sigma_{z z}$ is coupled to $\pi_{12}$. In $p$-type silicon this piezocoefficient is very small compared to the $\pi_{44}$ coefficient [6] resulting in a large uncertainty on the estimated $\sigma_{\mathrm{zz}}$ value. This problem can be eliminated by using a $n$-type piezoresistor, where the magnitudes of the piezocofficients are more comparable. $n$-type piezoresistors are currently being developed expecting realtime 3D stress determination of the epoxy curing process with a minimum uncertainty. Additionally, in $n$-type piezoresistors the $\sigma_{z z}$ pre- 


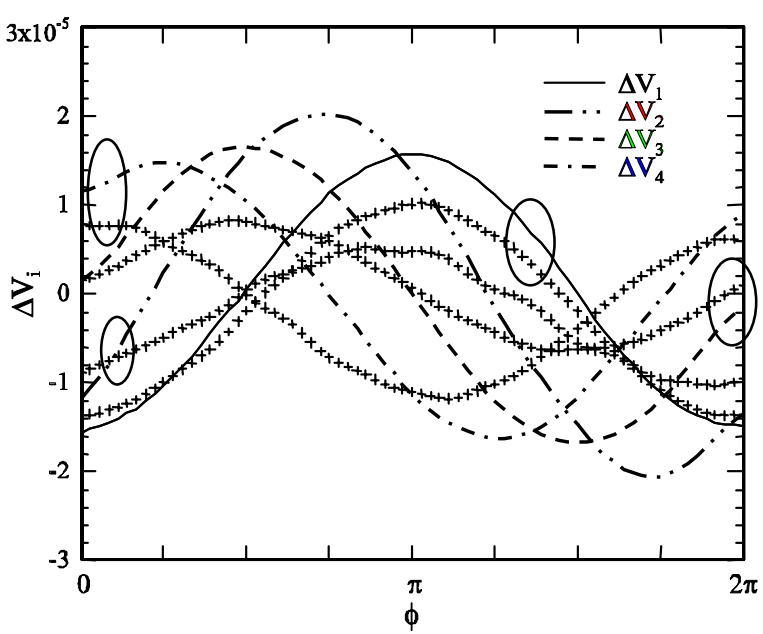

Figure 6. The change in voltage drop, $\Delta V_{i}$, where $i=1,2,3,4$, as a function of angle, $\phi$, for both a uniaxial stress of $\sigma_{x x}=11 \mathrm{MPa}$ (thin lines) and in package after curing (cross). The measured values of $V_{1}, V_{2}, V_{3}$, and $V_{4}$ are used in Eq. (1) to calculate $V_{p}$ and $V_{t}$.

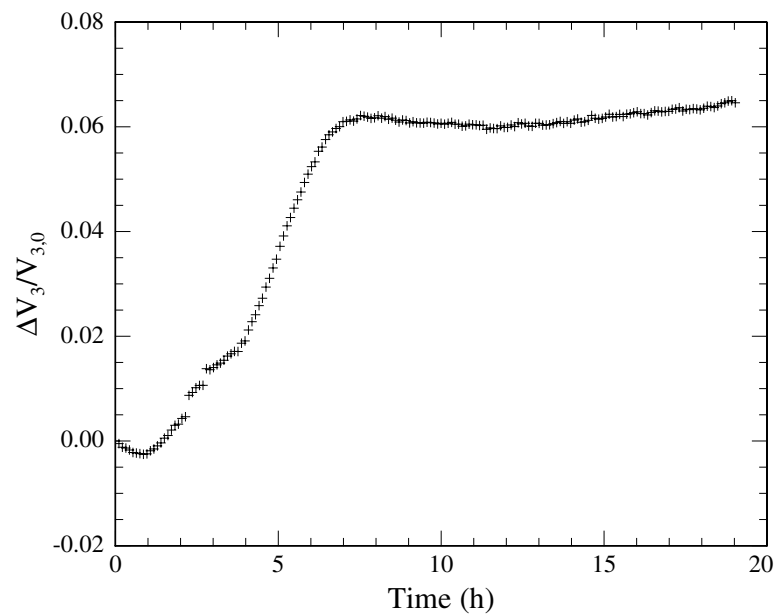

Figure 7. The relative change in voltage drop, $\Delta V_{3} / V_{3,0}$, as a function of time at the current angle $\phi=90^{\circ} . \Delta V_{3} / V_{3,0}$ for this angle is described by Eq. (4).

factor is $\mathrm{a}=2 \pi_{12} /\left(\pi_{11}+\pi_{12}\right) \approx-2$ and thus we are able to extract $\sigma_{z z}-\sigma_{y y}$ and equivalently $\sigma_{z z}-\sigma_{x x}$ with the use of a $n$-type piezoresistor.

\section{CONCLUSION}

In this paper we determine the stress distribution during an epoxy packaging curing process, adding

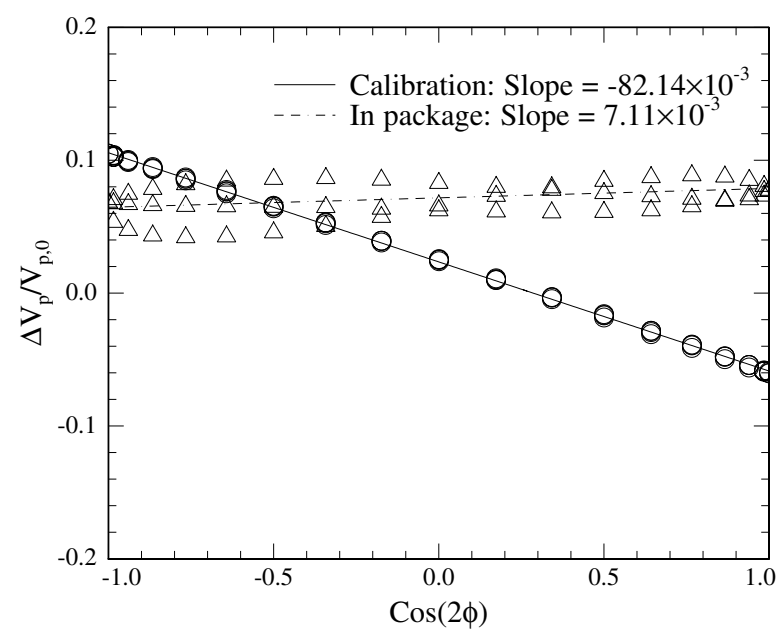

Figure 8. The fictive voltage drop $\Delta V_{p} / V_{p, 0}$ for both the calibration at a uniaxial stress of $77 \mathrm{MPa}$ (circular symbols) and in the package (triangle).

a new dimension to characterization of microsystem packaging. We expect that this method can be used in a wide range of packaging induced stress measurement applications. We can determine curing time with an uncalibrated sensor and using a calibrated sensor we can extract the stress distribution.

For the presented packaging concept using an Epotek 302-3M epoxy we find the stress in the package to be $\left(\sigma_{x x}-\sigma_{y y}\right)=6.7 \mathrm{MPa}$, and $\left(\sigma_{x x}+\sigma_{y y^{-}}\right.$ $\left.0.4 \sigma_{z z}\right)=232 \mathrm{MPa}$ and a curing time of 8 hours. Using $n$-type piezoresistors will lower the uncertainty of the extracted stress values and enable an extraction of $\sigma_{z z}-\sigma_{x x}$ and $\sigma_{z z}-\sigma_{y y}$.

\section{REFERENCES}

[1] P. Ruther, et al, Proc. IEEE Sensors 2006, pp. 314.

[2] A. Mian, J.C. Suhling, R.C. Jaeger, IEEE Sensors Journal, 6-2 (2006), pp. 340-356.

[3] A. Hyldgård, O. Hansen and E. V. Thomsen, Proc. IEEE MEMS 2005, pp. 303-306.

[4] J. Bartholomeyczik, et al, Proc. IEEE MEMS 2004, pp. 263-266.

[5] R. Steiner, et al, Sensors and Actuators A, 66 (1998), pp.167-172.

[6] C.S. Smith, Physical Review, 94 (1954), pp. 42-49.

[7] J. Richter, et al, Proc. IEEE MEMS 2007, pp. 69-72. 